\title{
A Bayesian nonparametric approach to testing for dependence between random variables Supplementary Material
}

\author{
Sarah Filippi* and Chris C. Holmes ${ }^{\dagger}$
}

\section{S1 Details on derivation of the Bayes Factor}

Under $\mathcal{M}_{0}$, the samples $x$ and $y$ are assumed to be independent; therefore

$$
\begin{aligned}
p\left(x, y \mid \Xi, \mathcal{C}, \mathcal{M}_{0}\right) & =p\left(x \mid \Xi_{X}, \mathcal{C}, \mathcal{M}_{0}\right) p\left(y \mid \Xi_{Y}, \mathcal{C}, \mathcal{M}_{0}\right) \\
& =\prod_{j} \xi_{j, X}^{n_{j 0}+n_{j 2}}\left(1-\xi_{j, X}\right)^{n_{j 1}+n_{j 3}} \prod_{j} \xi_{j, Y}^{n_{j 0}+n_{j 1}}\left(1-\xi_{j, Y}\right)^{n_{j 2}+n_{j 3}},
\end{aligned}
$$

where $\Xi_{X}=\left\{\xi_{j, X}\right\}_{j}$ and $\Xi_{Y}=\left\{\xi_{j, Y}\right\}_{j}$. Since we assumed that the random branching probabilities are independent and follow Beta distributions, $\xi_{j, X} \sim \operatorname{Beta}\left(\alpha_{j, X,(0)}, \alpha_{j, X,(1)}\right)$ and $\xi_{j, Y} \sim \operatorname{Beta}\left(\alpha_{j, Y,(0)}, \alpha_{j, Y,(1)}\right)$, then for all $j$,

$$
p\left(\xi_{j, X} \mid \mathcal{M}_{0}\right)=\frac{\xi_{j, X}^{\alpha_{j, X,(0)}-1}\left(1-\xi_{j, X}\right)^{\alpha_{j, X,(1)}-1}}{B\left(\alpha_{j, X,(0)}, \alpha_{j, X,(1)}\right)}
$$

where $B(.,$.$) denotes the Beta function. We obtain the marginal likelihood by integrating$ out the random branching probabilities as follows:

$$
\begin{aligned}
& p\left(x, y \mid \mathcal{C}, \mathcal{A}_{X}, \mathcal{A}_{Y}, \mathcal{M}_{0}\right)= \int p\left(x, y \mid \Xi, \mathcal{C}, \mathcal{A}_{X}, \mathcal{A}_{0}, \mathcal{M}_{0}\right) p\left(\Xi \mid \mathcal{M}_{0}\right) d \Xi \\
&= \prod_{j} \int \frac{\xi_{j, X}^{n_{j 0}+n_{j 2}+\alpha_{j, X,(0)}-1}\left(1-\xi_{j, X}\right)^{n_{j 1}+n_{j 3}+\alpha_{j, X,(1)}-1}}{B\left(\alpha_{j, X,(0)}, \alpha_{j, X,(1)}\right)} \\
&= \prod_{j} \frac{B\left(n_{j 0}+n_{j 2}+\alpha_{j, X,(0)}, n_{j 1}+n_{j 3}+\alpha_{j, X,(1)}\right)}{B\left(\alpha_{j, X,(0)}, \alpha_{j, X,(1)}\right)} \\
& \times \frac{\xi_{j, Y}^{n_{j 0}+n_{j 1}+\alpha_{j, Y,(0)}-1}\left(1-\xi_{j, X}\right)^{n_{j 2}+n_{j 3}+\alpha_{j, Y,(1)}-1}}{B\left(n_{j 0}+n_{j 1}+\alpha_{j, Y,(0)}, n_{j 2}+n_{j 3}+\alpha_{j, Y,(1)}\right)} \\
& B\left(\alpha_{j, Y,(0)}, \alpha_{j, Y,(1)}\right)
\end{aligned}
$$

Under hypothesis $\mathcal{M}_{1}$, we consider a probability vector of random branching prob-

\footnotetext{
* Department of Statistics, University of Oxford, England sarah.filippi@stats.ox.ac.uk

${ }^{\dagger}$ Department of Statistics and Oxford-Man Institute, University of Oxford, England cholmes@stats.ox.ac.uk
} 
abilities $\theta_{j}=\left(\theta_{j,(0)}, \theta_{j,(1)}, \theta_{j,(2)}, \theta_{j,(3)}\right) \in \mathbb{S}^{3}$ so that

$$
p\left(x, y \mid \Theta, \mathcal{C}, \mathcal{M}_{1}\right)=\prod_{j} \theta_{j,(0)}^{n_{j 0}} \theta_{j,(1)}^{n_{j 1}} \theta_{j,(2)}^{n_{j 2}} \theta_{j,(3)}^{n_{j 3}}
$$

where $\Theta=\left\{\theta_{j}\right\}_{j}$. Assuming that $\theta_{j}$ follows a Dirichlet distribution with parameters $\alpha_{j}=\left(\alpha_{j,(0)}, \alpha_{j,(1)}, \alpha_{j,(2)}, \alpha_{j,(3)}\right)$,

$$
p\left(\theta_{j} \mid \mathcal{M}_{1}\right)=\frac{\theta_{j,(0)}^{\alpha_{j,(0)}-1} \theta_{j,(1)}^{\alpha_{j,(1)}-1} \theta_{j,(2)}^{\alpha_{j,(2)}-1}\left(1-\theta_{j,(0)}-\theta_{j,(1)}-\theta_{j,(2)}\right)^{\alpha_{j,(3)}-1}}{\tilde{B}\left(\alpha_{j}\right)}
$$

where $\tilde{B}($.$) is the multinomial Beta function, which can be expressed in terms of the$ gamma function:

$$
\tilde{B}\left(\alpha_{j}\right)=\frac{\Gamma\left(\alpha_{j,(0)}\right) \Gamma\left(\alpha_{j,(1)}\right) \Gamma\left(\alpha_{j,(2)}\right) \Gamma\left(\alpha_{j,(3)}\right)}{\Gamma\left(\alpha_{j,(0)}+\alpha_{j,(1)}+\alpha_{j,(2)}+\alpha_{j,(3)}\right)} .
$$

Similarly to the $\mathcal{M}_{0}$ case, the marginal likelihood can be obtained by integrating out the random branching probabilities as follows:

$$
\begin{aligned}
p\left(x, y \mid \mathcal{C}, \mathcal{A}, \mathcal{M}_{1}\right) & =\int p\left(x, y \mid \Theta, \mathcal{C}, \mathcal{A}, \mathcal{M}_{1}\right) p\left(\Theta \mid \mathcal{M}_{1}\right) d \Theta \\
& =\prod_{j} \frac{1}{\tilde{B}\left(\alpha_{j}\right)} \int \prod_{i=0}^{3} \theta_{j i}^{\left(n_{j i}+\alpha_{j,(i)}-1\right)} d \theta_{j i}=\prod_{j} \frac{\tilde{B}\left(\tilde{n}_{j}+\alpha_{j}\right)}{\tilde{B}\left(\alpha_{j}\right)}
\end{aligned}
$$

where $\tilde{n}_{j}$ denotes the vector of counts of data: $\tilde{n}_{j}=\left(n_{j 0}, n_{j 1}, n_{j 2}, n_{j 3}\right)$.

To compute the Bayes factor we calculate the ratio of $p\left(x, y \mid \mathcal{M}_{0}\right)$ as defined in equation (1) over $p\left(x, y \mid \mathcal{M}_{1}\right)$ as defined in equation (3). Since both (1) and (3). can be written an infinite product over all possible sets, then the Bayes factor can also be written $\prod_{j} b_{j}$ where

$$
\begin{aligned}
b_{j}= & \frac{B\left(n_{j 0}+n_{j 2}+\alpha_{j, X,(0)}, n_{j 1}+n_{j 3}+\alpha_{j, X,(1)}\right)}{B\left(\alpha_{j, X,(0)}, \alpha_{j, X,(1)}\right)} \\
& \times \frac{B\left(n_{j 0}+n_{j 1}+\alpha_{j, Y,(0)}, n_{j 2}+n_{j 3}+\alpha_{j, Y,(1)}\right)}{B\left(\alpha_{j, Y,(0)}, \alpha_{j, Y,(1)}\right)} \times \frac{\tilde{B}\left(\alpha_{j}\right)}{B\left(\tilde{n}_{j}+\alpha_{j}\right)} .
\end{aligned}
$$

Expressing the Beta and the multinomial Beta function in terms of Gamma functions, we obtain

$$
\begin{aligned}
b_{j}= & \frac{\Gamma\left(n_{j 0}+n_{j 2}+2 a_{j}\right) \Gamma\left(n_{j 1}+n_{j 3}+2 a_{j}\right) \Gamma\left(n_{j 0}+n_{j 1}+2 a_{j}\right) \Gamma\left(n_{j 2}+n_{j 3}+2 a_{j}\right)}{\Gamma\left(n_{j 0}+n_{j 1}+n_{j 2}+n_{j 3}+4 a_{j}\right) \Gamma\left(n_{j 0}+a_{j}\right) \Gamma\left(n_{j 1}+a_{j}\right) \Gamma\left(n_{j 2}+a_{j}\right) \Gamma\left(n_{j 3}+a_{j}\right)} \\
& \times \frac{\Gamma\left(4 a_{j}\right) \Gamma\left(a_{j}\right)^{4}}{\Gamma\left(2 a_{j}\right)^{4}}
\end{aligned}
$$


It is easy to see that for any $j$ such that $n_{j 0}+n_{j 1}+n_{j 2}+n_{j 3}=0$,

$$
b_{j}=\frac{\Gamma\left(2 a_{j}\right)^{4} \Gamma\left(4 a_{j}\right) \Gamma\left(a_{j}\right)^{4}}{\Gamma\left(4 a_{j}\right) \Gamma\left(a_{j}\right)^{4} \Gamma\left(2 a_{j}\right)^{4}}=1 .
$$

In addition, if $n_{j 0}+n_{j 1}+n_{j 2}+n_{j 3}=1$, then

$$
\begin{aligned}
b_{j} & =\frac{\Gamma\left(1+2 a_{j}\right)^{2} \Gamma\left(2 a_{j}\right)^{2} \Gamma\left(4 a_{j}\right) \Gamma\left(a_{j}\right)^{4}}{\Gamma\left(1+4 a_{j}\right) \Gamma\left(1+a_{j}\right) \Gamma\left(a_{j}\right)^{3} \Gamma\left(2 a_{j}\right)^{4}} \\
& =\frac{\Gamma\left(1+2 a_{j}\right)^{2} \Gamma\left(4 a_{j}\right) \Gamma\left(a_{j}\right)}{\Gamma\left(1+4 a_{j}\right) \Gamma\left(1+a_{j}\right) \Gamma\left(2 a_{j}\right)^{2}} \\
& =\frac{\left(2 a_{j}\right)^{2} \Gamma\left(2 a_{j}\right)^{2} \Gamma\left(4 a_{j}\right) \Gamma\left(a_{j}\right)}{4 a_{j} \Gamma\left(4 a_{j}\right) a_{j} \Gamma\left(a_{j}\right) \Gamma\left(2 a_{j}\right)^{2}} \\
& =1
\end{aligned}
$$

where in the third line we use that for all $t, \Gamma(t+1)=t \Gamma(t)$.

\section{S2 Other approaches}

The square Pearson correlation is probably the most commonly used statistic to identify associations between two samples. This statistic accurately quantifies linear dependences but fails to detect dependencies when relationships are highly nonlinear. Another criteria, called mutual information (MI), arose from the field of information theory (Shannon and Weaver, 1949; Cover and Thomas, 1991). The original primary purpose of mutual information was to quantify bits of informations transmitted in a system. It has been used in a wide range of disciplines such as in economy (Maasoumi, 1993; Maasoumi and Racine, 2002), wireless security (Bloch et al., 2008), pattern analysis (Peng et al., 2005), neurobiology (Pereda et al., 2005) and systems biology (Cheong et al., 2011; Liepe et al., 2013; Uda et al., 2013; Mc Mahon et al., 2014). Mutual information is by definition a similarity measure between the joint probability of two variables and the product of the two marginal probability distributions; for the purposes of this paper considering continuous univariate random variables, $\{x, y\}$, MI is equivalent to the Kullback-Leibler divergence between the joint distribution and the product of marginal densities

$$
\mathrm{MI}=\int_{x, y} p(x, y) \log \left(\frac{p(x, y)}{p(x) p(y)}\right) d x d y .
$$

It is equal to 0 when the variables are independent and increases with the level of association of the two variables. Therefore, by extension, this measure has been employed to quantify dependencies between two variables. It is well-known that the estimation of the mutual information between two samples is not straightforward. It is often based on approximations of the probability distributions (Paninski, 2003; Cellucci et al., 2005; Khan et al., 2007) either using bin-based procedures, kernel densities (Moon et al., 1995), or k-nearest neighbours (Kraskov et al., 2004). 
Another classical approach for detecting dependencies between two continuous univariate random variables consists in partitioning the sample space into bins and evaluating non-parametric test statistics on the binned data (Heller et al., 2014). This type of approach includes the well known Hoeffding's test (Hoeffding, 1948) as well as the maximum information criterion (MIC) recently introduced by Reshef et al. (2011). In this last paper, the authors propose to estimate the mutual information using a bin-based procedure on any grid drawn on the scatterplot of the two variables up to a maximal grid precision. The MIC is then proportional to the highest mutual information on these grids. This recent work has lead to a series of discussion regarding properties that adequate measures of dependencies should fulfil (Gorfine et al., 2012; Simon and Tibshirani, 2014; Kinney and Atwal, 2014). More generally, dependences between multivariate random variables have commonly been modelled using copula transform (Hoff, 2007; Smith et al., 2012) or by introducing latent random variables (Petrone et al., 2009). For high-dimensional spaces, the problem of testing independence can also be formulated by embedding probability distributions into reproducing kernel Hilbert space (Gretton and Györfi, 2010). These papers provide innovative approaches to modelling dependence but do not provide a fully Bayesian nonparametric approach with an analytic Bayes factor for testing dependence vs independence, which is the focus of our paper here.

In the following, we consider 5 frequentist dependence statistics: the Rsquared measure, the distance correlation (dcor) (Székely et al., 2009), Hoeffding's D (Hoeffding, 1948), the mutual information estimated using the k-nearest neighbour method (Kraskov et al., 2004) and the maximum information criterion (MIC) (Reshef et al., 2011). We use the MATLAB implementation of these algorithms provided in the supplementary material of Kinney and Atwal (2014). The Rsquared measure, the distance correlation and Hoeffding'D methods detect higher associations between the variables for linear models; whereas MIC and MI detect highest dependencies for the sinusoidal and the circular models respectively (see Figure S1). In addition to its probabilistic properties, a crucial advantage of our Bayesian approach compared to these five other approaches is the interpretability of the measure of dependencies. Indeed, whenever the dependence statistic is higher than 0.5 there is, by definition, more evidence in favour of the dependence hypothesis than the independent one. In contrast, for all the frequentist methods identifying a threshold above which one can claim that two samples are dependent is a challenging task, as opposed to claiming evidence against the null. Typically, these thresholds are either chosen heuristically or calibrated via the construction of "null datasets" - by randomly permuting the indexes of the two samples in order to destroy potential dependence - and defining the threshold as a quantile of the distribution of the dependence statistics on these "null" datasets.

It is difficult to identify a measure that would allow us to fairly compare our Bayesian approach to more traditional frequentist approaches. A traditional frequentist measure of statistical test performance consists in computing the power of the test which measures the true positive rate (percentage of times the method detect dependences) for a given significance level (i.e. false positive rate). Here, for each dependence test, we chose a significance level of 0.05 , that is, we fix the detection threshold to be equal to the 0.95 quantile of the "null distribution" estimated via 500 permutations. In Figure $\mathrm{S} 2$, we observe that the power of every method strongly varies from one generative 


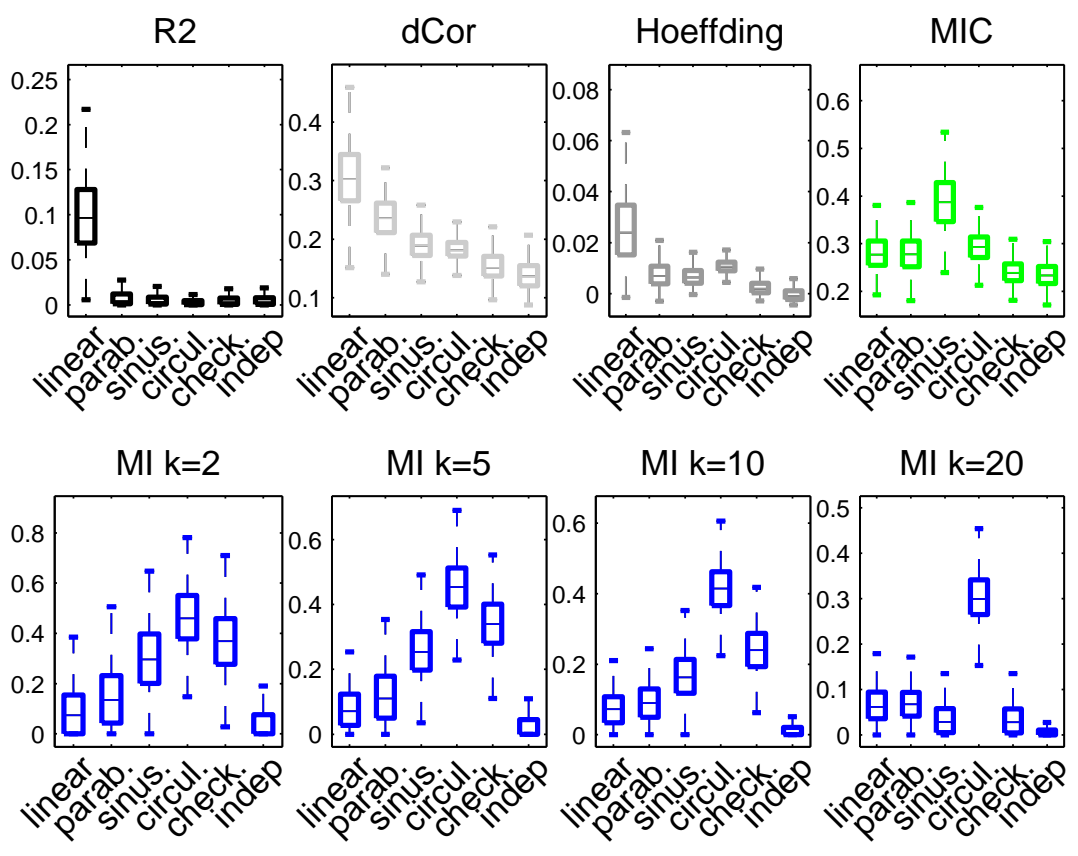

Figure S1: Distribution (over 500 independent runs) of the dependence measures quantified by the Rsquared correlation (black), the dCor (light grey) and the Hoeffding method (dark grey), the MIC (green) and the Mutual Information estimated with the k-nearest method for different values of $k$ (blue). Data are generated under the five illustrative examples as well as an independent generative model where both $x$ and $y$ are vectors of i.i.d. samples from a normal distribution with mean 0 and standard deviation 1. Here, $N=150$ and $\sigma=2$. 

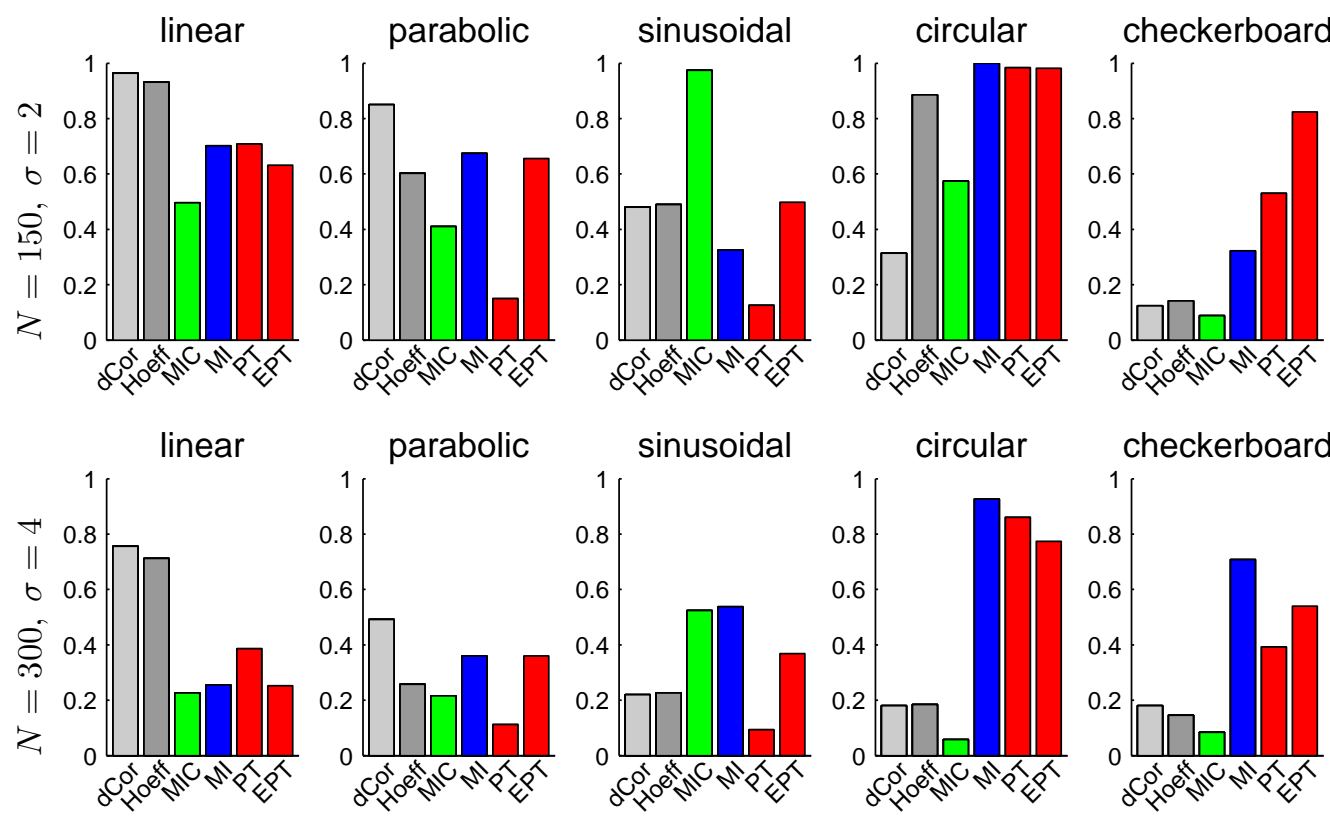

Figure S2: Power of each algorithm for each model for $N=150, \sigma=2$ (Top row) and $N=300, \sigma=4$ (Bottom row). The null distribution is computed via permutation; the significance level (i.e false positive rate) is set equal to 0.05. PT stands for Polya Tree and EPT designates the empirical version of our approach.

model to another. The power of the empirical Bayes version of our procedure (denoted by EPT in Figure S2) is comparable with that of the Mutual Information algorithm estimated using the 20-nearest neighbours. Further power analysis via ROC curves is shown in Figure S3. Using a threshold based on the "null distribution" is very atypical for Bayesian approaches as there exists a natural threshold for the probabilistic measure which is equal to 0.5 . Using this threshold the true positive and false positive rates for different generative models are summarized in the following table.

\begin{tabular}{|c|ccccc|c|}
\hline & \multicolumn{5}{|c|}{ True positive rate } & False positive \\
& linear & parab. & sinus. & circul. & check. & rate \\
\hline $\mathrm{PT}, N=150, \sigma=2$ & 0.82 & 0.31 & 0.33 & 1 & 0.82 & 0.13 \\
$\mathrm{EPT}, N=150, \sigma=2$ & 0.92 & 0.95 & 0.97 & 1 & 1 & 0.42 \\
\hline $\mathrm{PT}, N=300, \sigma=4$ & 0.45 & 0.17 & 0.21 & 0.91 & 0.56 & 0.09 \\
$\mathrm{EPT}, N=300, \sigma=4$ & 0.62 & 0.81 & 0.91 & 0.98 & 0.96 & 0.4 \\
\hline
\end{tabular}



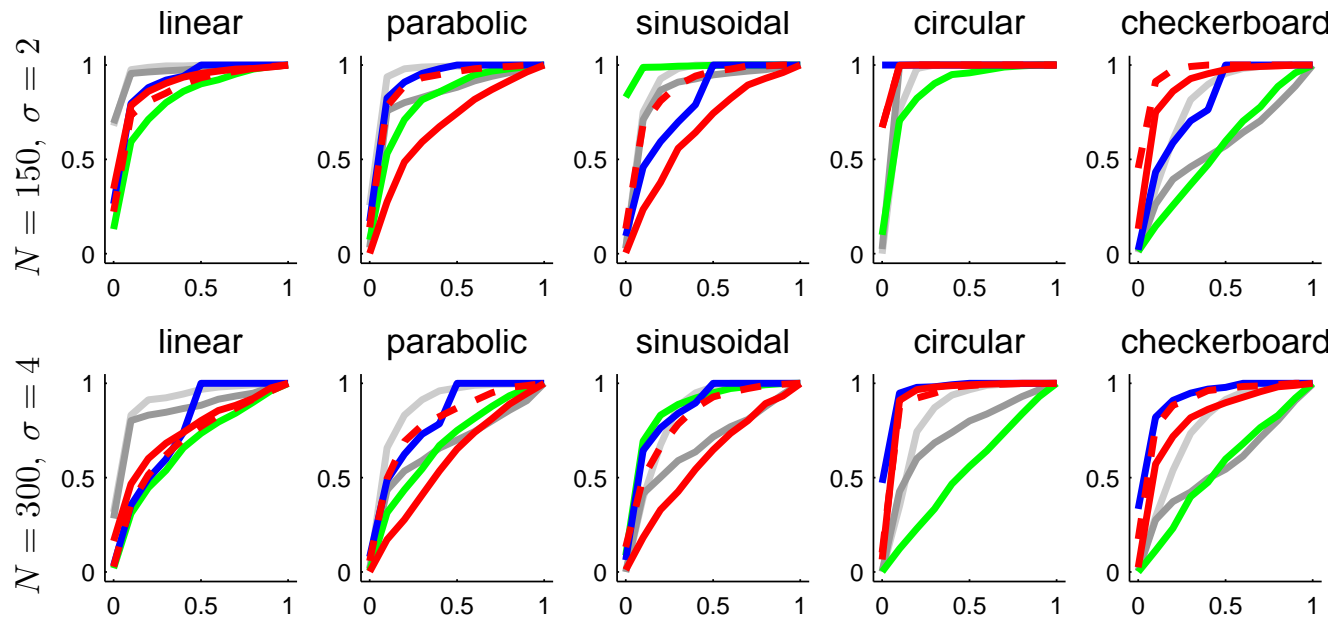

Figure S3: ROC curve illustrating the true positive rate as a function of the false positive rate for each model and each algorithm for $N=150, \sigma=2$ (Middle row) and $N=300$, $\sigma=4$ (Bottom row). The colour scheme is similar to the one from figure S1 except that the red curve represents our Bayesian nonparametric approach and the dashed red curve our approach using an empirical Bayes approach maximizing the marginal probability over the shifted partition scheme with data wrapping. For clarity, the ROC curve for Rsquared is not shown.

\section{S3 Additional figures}



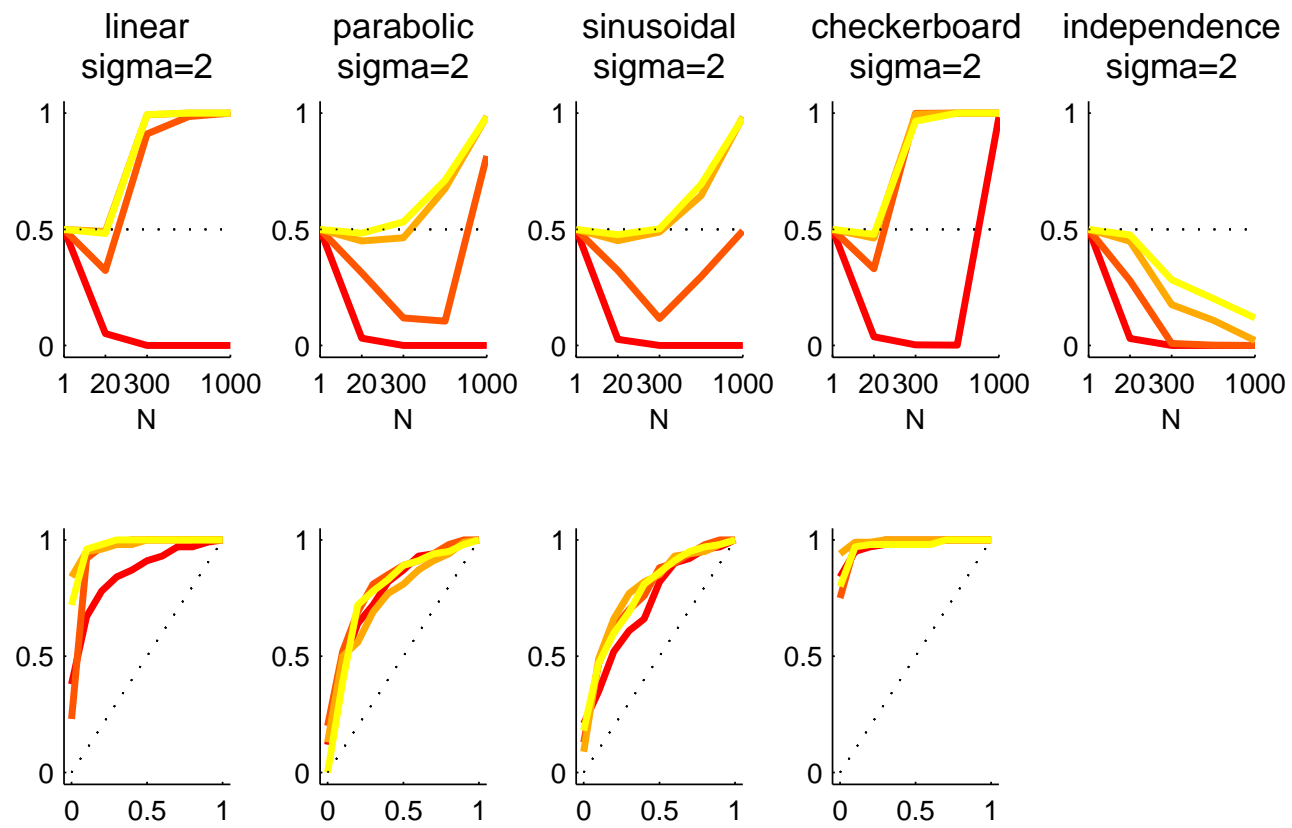

Figure S4: Impact of the parameter $c$ when $\sigma=2$. (Top) Median (over 500 runs of the probability of the dependent models as a function of the number of data points $(N)$ for different values of $c$. (Bottom) ROC curve for different values of $c$ when $N=300$. In both figure types, $\sigma=2$ and the color scheme represents different values of $c \in\{0.1,1,5,10\}$ where $c=0.1$ corresponds to the red line and $c=10$ corresponds to the yellow line. 

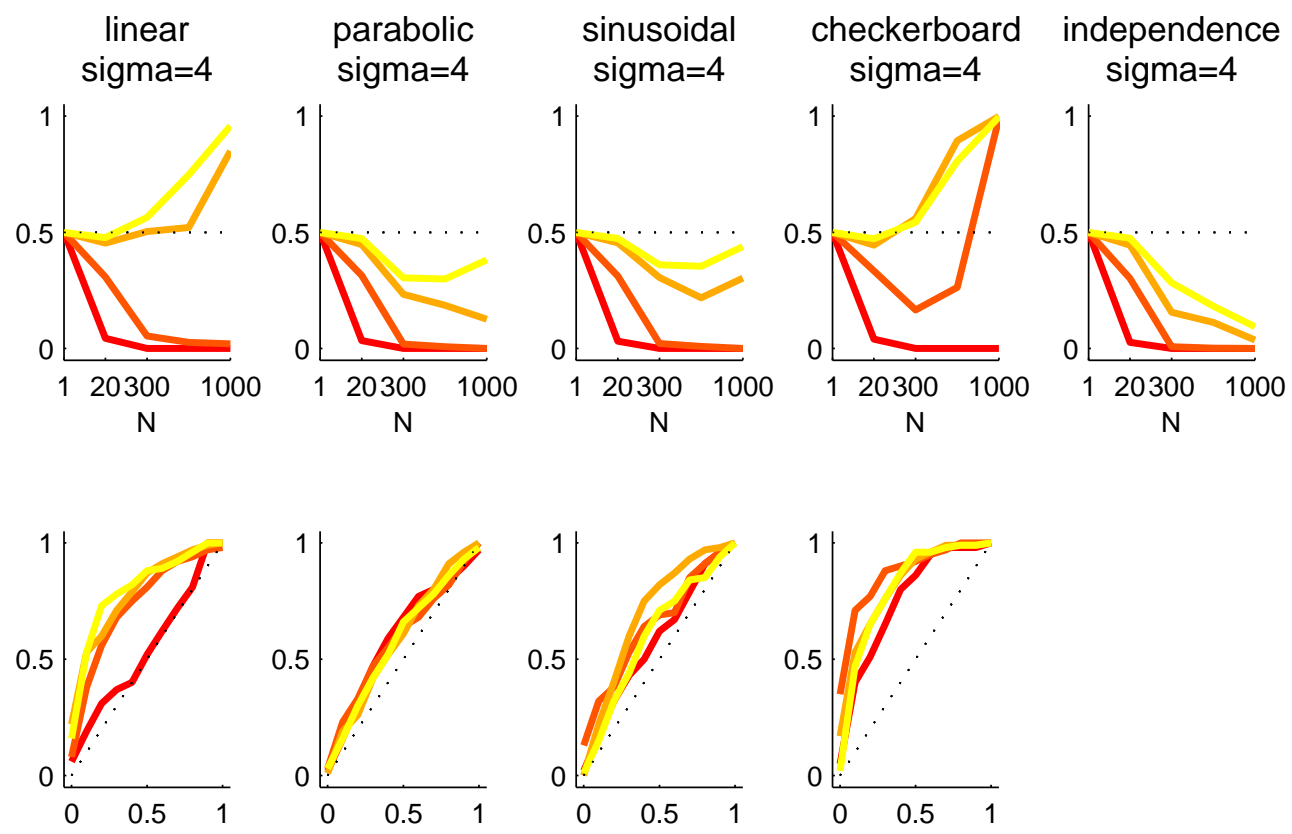

Figure S5: Impact of the parameter $c$ when $\sigma=4$. (Top) Median (over 500 runs of the probability of the dependent models as a function of the number of data points $(N)$ for different values of $c$. (Bottom) ROC curve for different values of $c$ when $N=300$. In both figure types, $\sigma=4$ and the color scheme represents different values of $c \in\{0.1,1,5,10\}$ where $c=0.1$ corresponds to the red line and $c=10$ corresponds to the yellow line. 


\section{References}

Bloch, M., Barros, J., Rodrigues, M. R., and McLaughlin, S. W. (2008). "Wireless information-theoretic security." Information Theory, IEEE Transactions on, 54(6): 2515-2534. 3

Cellucci, C., Albano, A. M., and Rapp, P. (2005). "Statistical validation of mutual information calculations: Comparison of alternative numerical algorithms." Physical Review E, 71(6): 066208. 3

Cheong, R., Rhee, A., Wang, C. J., Nemenman, I., and Levchenko, A. (2011). "Information transduction capacity of noisy biochemical signaling networks." Science, 334(6054): 354-358. 3

Cover, T. M. and Thomas, J. (1991). "Elements of information." TheoryWiley, New York. 3

Gorfine, M., Heller, R., and Heller, Y. (2012). "Comment on detecting novel associations in large data sets." Unpublished manuscript, http://iew3.technion.ac.il/gorfinm/files/science6.pdf. 4

Gretton, A. and Györfi, L. (2010). "Consistent nonparametric tests of independence." The Journal of Machine Learning Research, 11: 1391-1423. 4

Heller, R., Heller, Y., Kaufman, S., Brill, B., and Gorfine, M. (2014). "Consistent distribution-free $K$-sample and independence tests for univariate random variables." arXiv preprint arXiv:1410.6758. 3

Hoeffding, W. (1948). "A non-parametric test of independence." The Annals of Mathematical Statistics, 546-557. 3, 4

Hoff, P. D. (2007). "Extending the rank likelihood for semiparametric copula estimation." The Annals of Applied Statistics, 265-283. 4

Khan, S., Bandyopadhyay, S., Ganguly, A. R., Saigal, S., Erickson III, D. J., Protopopescu, V., and Ostrouchov, G. (2007). "Relative performance of mutual information estimation methods for quantifying the dependence among short and noisy data." Physical Review E, 76(2): 026209. 3

Kinney, J. B. and Atwal, G. S. (2014). "Equitability, mutual information, and the maximal information coefficient." Proceedings of the National Academy of Sciences, 111(9): 3354-3359. 4

Kraskov, A., Stögbauer, H., and Grassberger, P. (2004). "Estimating mutual information." Physical review E, 69(6): 066138. 3, 4

Liepe, J., Filippi, S., Komorowski, M., and Stumpf, M. P. (2013). "Maximizing the information content of experiments in systems biology." PLoS computational biology, 9(1): e1002888. 3

Maasoumi, E. (1993). "A compendium to information theory in economics and econometrics." Econometric reviews, 12(2): 137-181. 3 
Maasoumi, E. and Racine, J. (2002). "Entropy and predictability of stock market returns." Journal of Econometrics, 107(1): 291-312. 3

Mc Mahon, S. S., Sim, A., Filippi, S., Johnson, R., Liepe, J., Smith, D., and Stumpf, M. P. (2014). "Information theory and signal transduction systems: From molecular information processing to network inference." In Seminars in cell $\&$ developmental biology, volume 35, 98-108. Elsevier. 3

Moon, Y.-I., Rajagopalan, B., and Lall, U. (1995). "Estimation of mutual information using kernel density estimators." Physical Review E, 52(3): 2318. 3

Paninski, L. (2003). "Estimation of entropy and mutual information." Neural Computation, 15(6): 1191-1253. 3

Peng, H., Long, F., and Ding, C. (2005). "Feature selection based on mutual information criteria of max-dependency, max-relevance, and min-redundancy." Pattern Analysis and Machine Intelligence, IEEE Transactions on, 27(8): 1226-1238. 3

Pereda, E., Quiroga, R. Q., and Bhattacharya, J. (2005). "Nonlinear multivariate analysis of neurophysiological signals." Progress in neurobiology, 77(1): 1-37. 3

Petrone, S., Guindani, M., and Gelfand, A. E. (2009). "Hybrid Dirichlet mixture models for functional data." Journal of the Royal Statistical Society: Series B (Statistical Methodology), 71(4): 755-782. 4

Reshef, D. N., Reshef, Y. A., Finucane, H. K., Grossman, S. R., McVean, G., Turnbaugh, P. J., Lander, E. S., Mitzenmacher, M., and Sabeti, P. C. (2011). "Detecting novel associations in large data sets." science, 334(6062): 1518-1524. 3, 4

Shannon, C. E. and Weaver, W. (1949). "The mathematical theory of information." 3

Simon, N. and Tibshirani, R. (2014). "Comment on" Detecting Novel Associations In Large Data Sets" by Reshef Et Al, Science Dec 16, 2011." arXiv preprint arXiv:1401.7645. 4

Smith, M. S., Gan, Q., and Kohn, R. J. (2012). "Modelling dependence using skew t copulas: Bayesian inference and applications." Journal of Applied Econometrics, 27(3): 500-522. 4

Székely, G. J., Rizzo, M. L., et al. (2009). "Brownian distance covariance." The annals of applied statistics, 3(4): 1236-1265. 4

Uda, S., Saito, T. H., Kudo, T., Kokaji, T., Tsuchiya, T., Kubota, H., Komori, Y., Ozaki, Y.-i., and Kuroda, S. (2013). "Robustness and compensation of information transmission of signaling pathways." Science, 341(6145): 558-561. 3 